\title{
Continuous capnography monitoring during resuscitation in a transitional large mammalian model of asphyxial cardiac arrest
}

Praveen Chandrasekharan', Payam Vali², Munmun Rawat ${ }^{1}$, Bobby Mathew ${ }^{1}$, Sylvia F. Gugino' ${ }^{1}$, Carmon Koenigsknecht ${ }^{1}$, Justin Helman', Jayasree Nair', Sara Berkelhamer ${ }^{1}$ and Satyan Lakshminrusimha'

BACKGROUND: In neonates requiring chest compression (CC) during resuscitation, neonatal resuscitation program (NRP) recommends against relying on a single feedback device such as end-tidal carbon dioxide $\left(\mathrm{ETCO}_{2}\right)$ or saturations $\left(\mathrm{SpO}_{2}\right)$ to determine return of spontaneous circulation (ROSC) until more evidence becomes available.

METHODS: We evaluated the role of monitoring $\mathrm{ETCO}_{2}$ during resuscitation in a lamb model of cardiac arrest induced by umbilical cord occlusion $(n=21)$. Lambs were resuscitated as per NRP guidelines. Systolic blood pressure (SBP), carotid and pulmonary blood flows along with $\mathrm{ETCO}_{2}$ and blood gases were continuously monitored. Resuscitation was continued for 20 min or until ROSC (whichever was earlier). Adequate CC was arbitrarily defined as generation of $30 \mathrm{mmHg}$ SBP during resuscitation. $\mathrm{ETCO}_{2}$ thresholds to predict adequacy of CC and detect ROSC were determined.

RESULTS: Significant relationship between $\mathrm{ETCO}_{2}$ and adequate CC was noted during resuscitation (AUC-0.735, $P<0.01$ ). At $\operatorname{ROSC}(n=12), \mathrm{ETCO}_{2}$ rapidly increased to $57 \pm 20 \mathrm{mmHg}$ with a threshold of $\geq 32 \mathrm{mmHg}$ being $100 \%$ sensitive and $97 \%$ specific to predict ROSC.

CONCLUSION: In a large mammalian model of perinatal asphyxia, continuous $\mathrm{ETCO}_{2}$ monitoring predicted adequacy of $\mathrm{CC}$ and detected ROSC. These findings suggest $\mathrm{ETCO}_{2}$ in conjunction with other devices may be beneficial during $\mathrm{CC}$ and predict ROSC.

V entilation of the lungs is the most effective action in neonatal resuscitation. In severely asphyxiated infants, chest compressions are indicated if profound bradycardia (heart rate $<60 / \mathrm{min}$ ) or asystole persist despite adequate ventilation (1-4). The initiation of effective ventilation and chest compressions establishes blood flow through the lungs leading to gas exchange and expulsion of $\mathrm{CO}_{2}$ (5). Successful resuscitation will result in return of spontaneous circulation (ROSC). At present, there are no feedback devices approved by the neonatal resuscitation program (NRP) to assess adequacy of chest compressions and detect ROSC. As a result, chest compressions are frequently interrupted to assess heart rate and detect ROSC. Interruption of chest compressions delays the time to attain ROSC and can compromise outcomes $(6,7)$. Studies have shown that clinical assessment of ventilation and chest compressions during resuscitation are highly subjective and inaccurate with significant variability between observers $(8,9)$. Use of a noninvasive feedback device during resuscitation may address these limitations and assist in optimizing care and improving outcomes. An ideal feedback device when used during neonatal resuscitation may accurately assess effective ventilation and chest compressions, minimize hands off time during resuscitation and predict ROSC. The new guidelines recommend electrocardiography (EKG) monitoring during chest compressions. EKG monitoring will enhance our ability to detect ROSC but has two disadvantages - the pattern is likely to be distorted during chest compressions and it does not assess adequacy of chest compressions.

The purpose of performing chest compressions is to generate antegrade blood flow by increasing intravascular pressure (10). In this study, we used systolic blood pressure (SBP) achieved during the compression phase as a marker of adequacy of chest compressions.

We have previously demonstrated that use of capnography in addition to arterial carbon dioxide $\left(\mathrm{PaCO}_{2}\right)$ monitoring in a term lamb model of meconium aspiration resulted in maintenance of carbon dioxide in a narrower range and prevented fluctuation of carotid blood flow (11). From our pilot studies, using an asphyxiated cardiac arrest model in which active resuscitation was uninterrupted, we noted that changes in end-tidal carbon dioxide $\left(\mathrm{ETCO}_{2}\right)$ reflected gas exchange and also helped predict ROSC. In addition, following resuscitation, avoiding extremes of $\mathrm{PaCO}_{2}$ are important. Asphyxia is commonly associated with persistent pulmonary hypertension of the newborn (PPHN) and high $\mathrm{PaCO}_{2}$ is associated with acidosis and increase pulmonary vascular resistance (12). Hypocapnia can reduce cerebral blood flow and is associated with poor neurodevelopmental outcomes in infants with $\mathrm{HIE}$ (13). Monitoring $\mathrm{ETCO}_{2}$ can potentially avoid fluctuations in $\mathrm{PaCO}_{2}$ during the postresuscitation phase. 
Based on these observations, our study aimed to see if capnography can be used as a feedback device during resuscitation. Our objectives were to study if (i) end-tidal $\left(\mathrm{ETCO}_{2}\right)$ or expired $\mathrm{CO}_{2}$ can be used to predict adequacy of chest compressions during resuscitation, (ii) $\mathrm{ETCO}_{2}$ can predict ROSC, and (iii) $\mathrm{ETCO}_{2}$ and arterial carbon dioxide correlated postresuscitation.

\section{METHODS}

This study was approved by the University at Buffalo Institutional Animal Care and Use Committee (IACUC). Time-dated pregnant ewes from May Farms (Buffalo Mills, PA) were fasted overnight and underwent cesarean section under ketamine, diazepam sedation and $2 \%$ isoflurane anesthesia.

\section{Instrumentation}

Under maternal anesthesia, term lambs (139-141 d) were partially exteriorized and instrumented while on placental circulation. Jugular venous and carotid lines were placed for intravenous access and blood draws. Carotid and pulmonary flow probes (Transonic systems, Ithaca, $\mathrm{NY}$ ) were used to measure flows. Low lying umbilical arterial catheter was placed for transducing blood pressures. After instrumentation, the chest was closed in layers. The lambs were intubated and lung liquid drained. Cord occlusion resulted in asphyxia and cardiac arrest. Resuscitation was initiated after $5 \mathrm{~min}$ of cardiac arrest (Heart rate $=0$ for $5 \mathrm{~min}$ by arterial tracing and EKG) and as per NRP protocol.

\section{Resuscitation Protocol}

Five NRP providers with designated roles were present at each delivery. The providers performed the following roles: (i) lead and direct resuscitation, (ii) provide PPV using a T-piece resuscitator, (iii) provide chest compressions, (iv) administer epinephrine, and (v) record events. Two research assistants drew blood gases and analyzed them at the bedside. An $\mathrm{ETCO}_{2}$ adapter was attached to the endotracheal tube (ETT) and lambs were ventilated with a T-piece resuscitator. We used a Philips NM3 monitor (Respironics, MA) with flow parameters.

Resuscitation was initiated with $30 \mathrm{~s}$ of effective PPV with peak inspiratory pressures of $35 \mathrm{cmH}_{2} \mathrm{O}$ and PEEP of $5 \mathrm{cmH}_{2} \mathrm{O}$ with $21 \%$ $\mathrm{O}_{2}$. If there was no ROSC, chest compressions were initiated with a 3:1 compression to ventilation ratio and inspired oxygen concentration was increased to $100 \%$. Epinephrine $(0.03 \mathrm{mg} / \mathrm{kg}, 1: 10,000$ concentrations, $0.3 \mathrm{ml} / \mathrm{kg}$ ) was administered at 3 - min intervals until ROSC through an intravenous line. ROSC was defined as a sustained heart rate $>60 / \mathrm{min}$ with SBP of $\geq 30 \mathrm{mmHg}$. Resuscitation was continued until ROSC or until 20 min from onset of PPV (whichever was earlier).

PPV was initiated uniformly with pressures of $35 / 5 \mathrm{cmH}_{2} \mathrm{O}$ with tidal volumes of $7-8 \mathrm{ml} / \mathrm{kg}$. During resuscitation, in our study, effective SBP was defined as $50 \%$ of baseline systemic pressures prior to asphyxia which was approximately $\geq 30 \mathrm{mmHg}$. Arterial blood gases were collected and analyzed every minute until $20 \mathrm{~min}$. The data were continuously collected by BIOPAC Systems (Goleta, CA) software version - 4.3.1.

\section{Statistics}

Ordinal data were analyzed using $\chi^{2}$-test while continuous data were analyzed using unpaired $t$-test. Mann-Whitney $U$-test was used as an alternative nonparametric test where applicable. Sensitivity and specificity analysis with receiver operator curve was generated for $\mathrm{ETCO}_{2}$ to predict effective chest compressions ( $\mathrm{SBP} \geq 30 \mathrm{mmHg}$ ) during resuscitation and $\mathrm{ETCO}_{2}$ to predict ROSC. A plot of the true positives against the false positives for different test cut-off points were plotted as receiver operator curve. The cut-off points were assessed based on area under the curve (AUC), accuracy and the best sensitivity/specificity. Pearson correlation analysis post ROSC for $\mathrm{ETCO}_{2}$ and $\mathrm{PaCO}_{2}$ was performed. Bland-Altman plot was used to analyze agreement between $\mathrm{PaCO}_{2}$ and $\mathrm{ETCO}_{2}$. Box plots for all $\mathrm{ETCO}_{2}$ values for $\mathrm{SBP} \geq$ $30 \mathrm{mmHg}$ and for ROSC vs. no ROSC were generated. Data were analyzed using SPSS 22 software (IBM, NY) and prediction analysis was done using XLSTAT (Addinsoft, NY). Probability of $<5 \%$ was used for statistical significance.

\section{RESULTS}

We studied 21 term asphyxiated lambs. Baseline characteristics such as birth weight, gender, lung liquid volume, and blood gas prior to resuscitation are shown in Table 1. Following resuscitation with positive pressure ventilation (PPV), chest compressions and epinephrine, 12 lambs achieved ROSC while 9 did not achieve ROSC. Comparison of the characteristics between the lambs that achieved ROSC and the ones that did not, demonstrated no significant difference in baseline $\mathrm{pH}, \mathrm{PaCO}_{2}$, base excess or difference in time to arrest, volume of lung liquid, gender or weight (Table 1). The average time taken to achieve ROSC in those who survived was $4.9 \pm 3 \mathrm{~min}$.

\section{Ventilation Parameters and Initial $\mathrm{ETCO}_{2}$}

Tidal volumes generated by PPV for the first $5 \mathrm{~min}$ are shown in Figure 1. The mean peak inspiratory pressures were constant at $35 \pm 2.1 \mathrm{cmH}_{2} \mathrm{O}$ which resulted in a tidal volume of $1.8 \pm 0.5 \mathrm{ml} / \mathrm{kg}$ in the first minute and $6.9 \pm 0.4 \mathrm{ml} / \mathrm{kg}$ by $5 \mathrm{~min}$. At the time of initiation of ventilation, initial $\mathrm{ETCO}_{2}$ values (residual $\mathrm{CO}_{2}$ - Figure 2a) were 20 (IQR 10-30) mmHg compared with the remainder of the time where $\mathrm{ETCO}_{2}$ values were 15 (IQR 12-22) $\mathrm{mmHg}$ prior to ROSC or at the end of resuscitation which was significantly different $(P<0.01)$.

Table 1. Baseline characteristics

\begin{tabular}{lcc}
\hline Parameters (mean $\pm \mathrm{SD})$ & $\mathrm{ROSC}(\mathrm{N}=12)$ & No ROSC $(N=9)$ \\
\hline $\mathrm{pH}$ & $6.87 \pm 0.05$ & $6.83 \pm 0.07$ \\
$\mathrm{PaCO}_{2}(\mathrm{mmHg})$ & $135.7 \pm 13.9$ & $123.1 \pm 18.7$ \\
Base excess & $-16.1 \pm 1.9$ & $-17.8 \pm 2.9$ \\
Time to arrest (minutes) & $10.9 \pm 5$ & $10.5 \pm 5$ \\
Lung liquid $(\mathrm{ml})$ & $86 \pm 23$ & $71 \pm 23$ \\
Male (\%) & $9(75)$ & $7(77)$ \\
Birth weight $(\mathrm{kg})$ & $4.0 \pm 0.9$ & $3.8 \pm 0.7$ \\
\hline
\end{tabular}

Return of spontaneous circulation (ROSC) time average $4.9 \pm 3 \mathrm{~min}$.

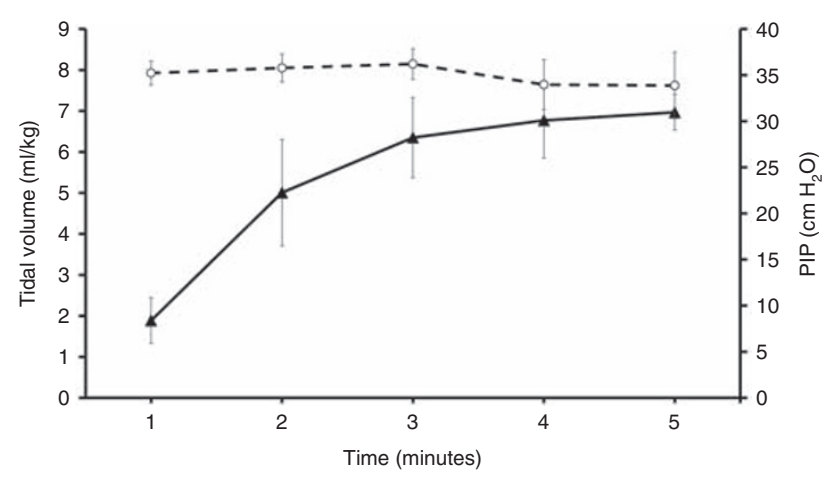

Figure 1. Respiratory parameters: Changes in tidal volume and peak inspiratory pressure in the first $5 \mathrm{~min}$ of resuscitation. The $\mathrm{x}$-axis represents the time points in minutes. The primary $y$-axis represents the tidal volume (black triangle) in $\mathrm{ml} / \mathrm{kg}$. Secondary y-axis represents the peak inspiratory pressure (circles) (PIP) in $\mathrm{cm} \mathrm{H}_{2} \mathrm{O}$. 


\section{Articles $\quad$ Chandrasekharan et al.}

a

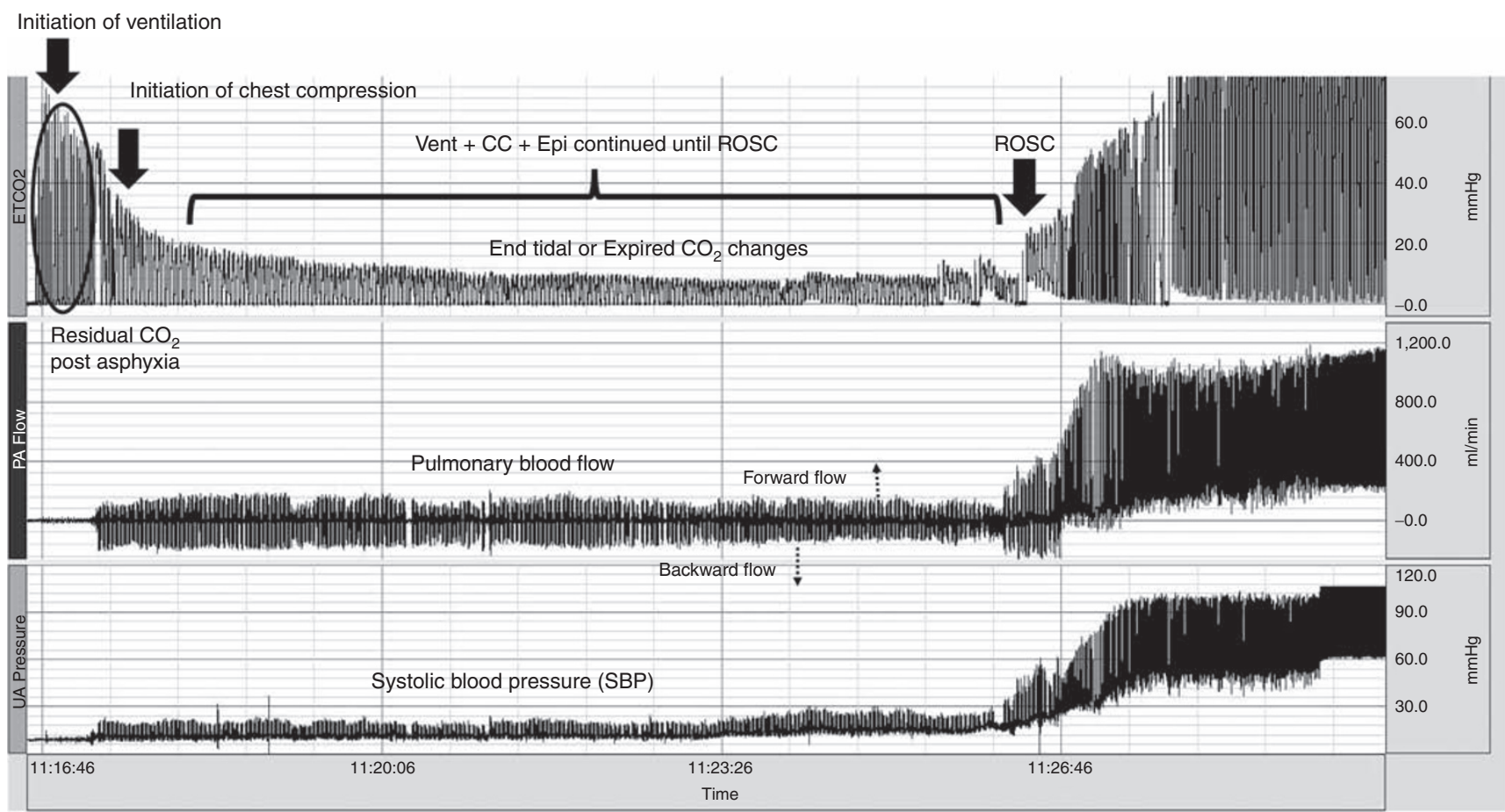

b

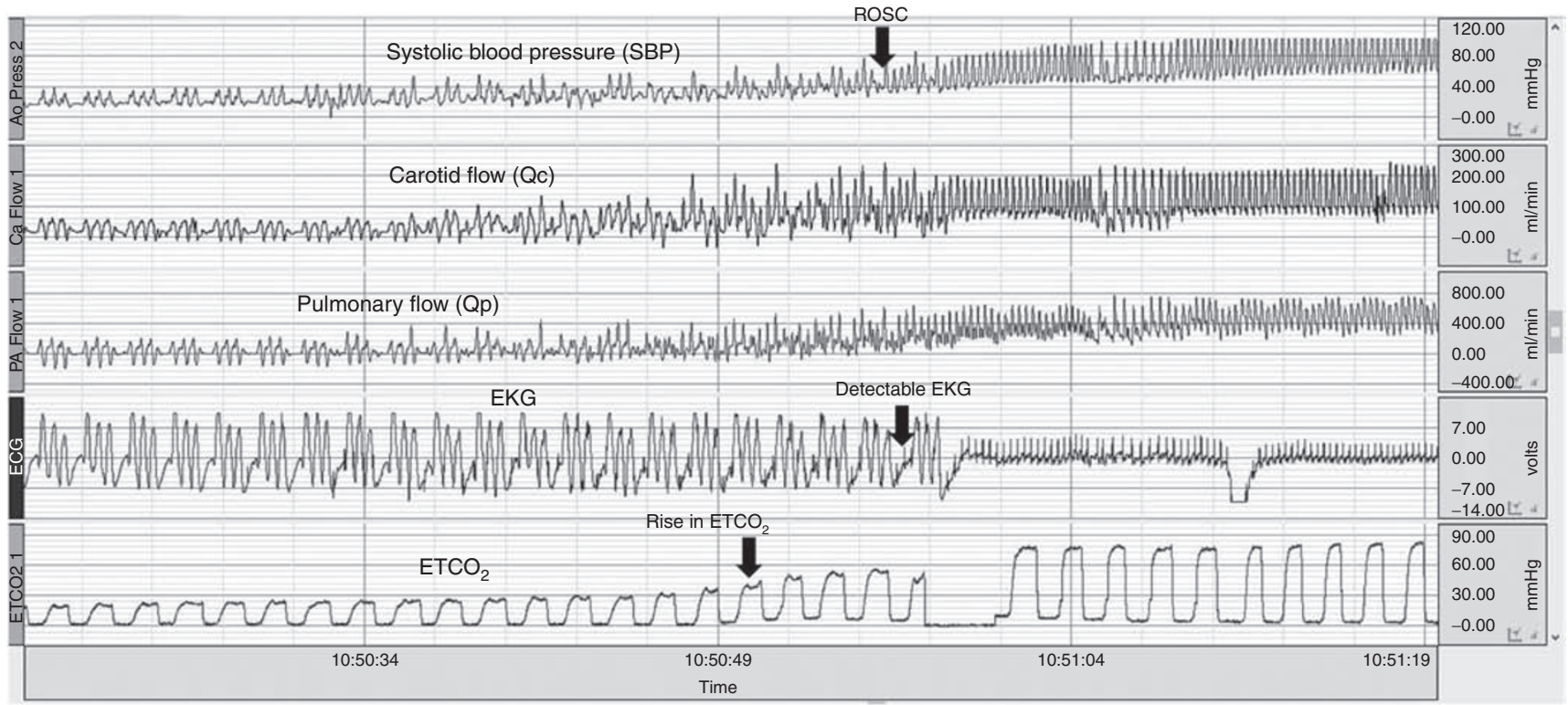

Figure 2. BIOPAC snapshot. (a) A representative snapshot of BIOPAC image showing changes in $\mathrm{ETCO}_{2}$, pulmonary blood flow and systolic blood pressure during resuscitation. Changes occurring with ventilation, $\mathrm{CC}$, and $\mathrm{ROSC}$ are shown with downward pointing arrows. Residual $\mathrm{CO}_{2}$ is represented by black circle. Parenthesis denotes active resuscitation. Hyphenated arrow represents positive \& negative pulmonary flow during CC. (b) A representative snapshot around the time of ROSC showing systolic blood pressure, carotid blood flow, pulmonary blood flow, EKG, and ETCO ${ }_{2}$. Downward arrow points at ROSC, detections of EKG and rise in $\mathrm{ETCO}_{2}$. CC, chest compression; $\mathrm{ETCO}_{2^{\prime}}$ end-tidal carbon dioxide; ROSC, return of spontaneous circulation.

Assessment of Adequacy of Chest Compressions (ETCO 2 and SBP $\geq 30 \mathrm{mmHg}$ )

We subsequently compared $\mathrm{ETCO}_{2}$ during chest compressions with systolic pressure generated by an umbilical arterial catheter with its tip in the ascending aorta. The ability of $\mathrm{ETCO}_{2}$ values to detect $\mathrm{SBP} \geq 30 \mathrm{mmHg}$ are tabulated with different sensitivities, specificities, positive predictive value (PPV) and negative predictive value (NPV) in Table 2. Receiver operator curve for $\mathrm{ETCO}_{2}$ and SBP are shown (Figure 3a). The AUC was 0.735 (confidence interval (CI): 0.66-0.82, $P<0.01$ ) for $\mathrm{ETCO}_{2}$ of $15 \mathrm{mmHg}$ to predict $\mathrm{SBP} \geq 30 \mathrm{mmHg}$. The $\mathrm{ETCO}_{2}$ values (12 (IQR 8-15) mmHg) associated with SBP < $30 \mathrm{mmHg}$ were significantly lower than $\mathrm{ETCO}_{2}(18$ (IQR 14-22) mmHg)) associated with SBP $\geq 30 \mathrm{~mm} \mathrm{Hg}$ (Figure $3 \mathbf{b})(P<0.01)$. 


\begin{tabular}{lccccc}
\hline $\begin{array}{l}\mathrm{ETCO}_{2} \\
(\mathrm{mmHg})\end{array}$ & Sensitivity & Specificity & PPV & NPV & Accuracy \\
\hline 11 & $96 \%$ & $30 \%$ & $56 \%$ & $89 \%$ & $63 \%$ \\
13 & $84 \%$ & $49 \%$ & $60 \%$ & $70 \%$ & $65 \%$ \\
15 & $69 \%$ & $66 \%$ & $65 \%$ & $70 \%$ & $67 \%$ \\
17 & $60 \%$ & $76 \%$ & $70 \%$ & $67 \%$ & $69 \%$ \\
19 & $49 \%$ & $83 \%$ & $72 \%$ & $64 \%$ & $67 \%$ \\
\hline
\end{tabular}

$\mathrm{ETCO}_{2}$, end-tidal carbon dioxide; NPV, negative predictive value; PPV, positive predictive value; ROSC. return of spontaneous circulation; $\mathrm{SBP}$, systolic blood pressure.

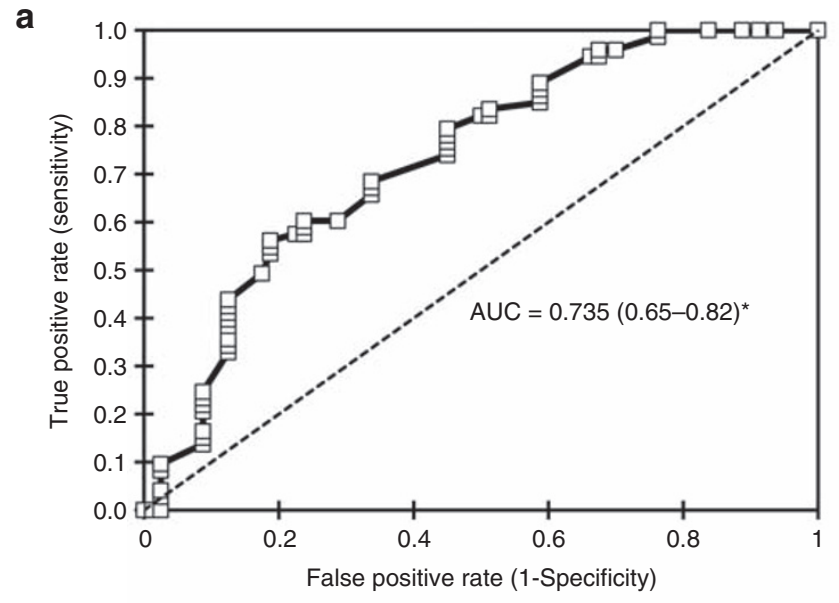

b

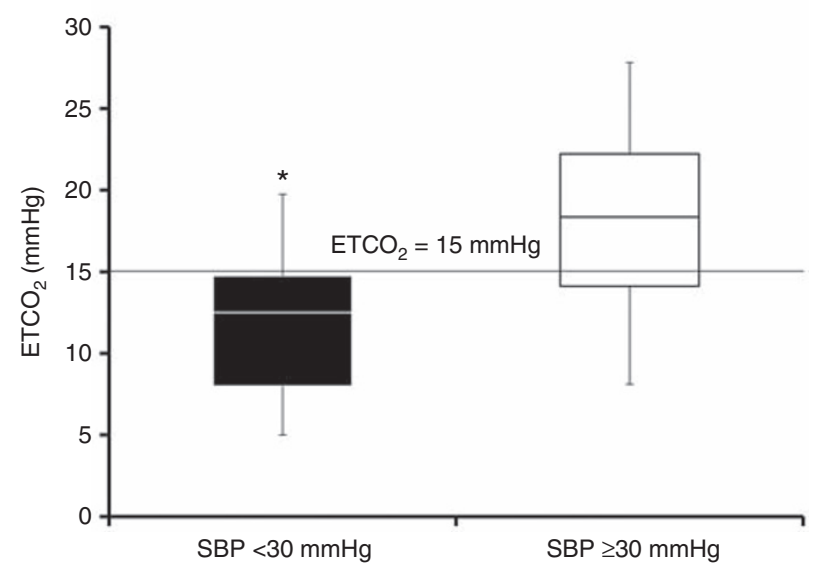

Figure 3. Prediction analysis ETCO 2 and SBP. (a) Receiver operator curve for end-tidal $\mathrm{CO}_{2}\left(\mathrm{ETCO}_{2}\right)$ to predict systolic blood pressure $\geq 30 \mathrm{mmHg}$. (b) Box plots of all ETCO values $(\mathrm{mmHg})$ associated with systolic blood pressure of $<30$ (black box plot) and $\geq 30 \mathrm{mmHg}$ (white box plot). SBP = systolic blood pressure. ${ }^{*}$ denotes a statistical significance $P<0.01$.

During chest compressions, SBP $\geq 30 \mathrm{~mm}$ Hg predicted left carotid arterial blood flow of $3.2 \mathrm{ml} / \mathrm{kg} / \mathrm{min}$ with AUC of 0.85 (CI: $0.75-0.96, P<0.01)$.

\section{$\mathrm{ETCO}_{2}$ and ROSC}

The $\mathrm{ETCO}_{2}$ values for predicting ROSC are shown in Table 3. The AUC was 0.990 for $\mathrm{ETCO}_{2}$ of $32 \mathrm{mmHg}$ to predict ROSC (Figure 4a). The $\mathrm{ETCO}_{2}$ values before (17 (IQR 11-25) $\mathrm{mmHg}$ ) and after (66 (IQR 59-72) mmHg) ROSC are shown in Figure $4 \mathbf{b}$ which were significantly different $(P<0.01)$.
Table 3. $\mathrm{ETCO}_{2}$ values to predict ROSC

\begin{tabular}{lccccc}
\hline $\begin{array}{l}\mathrm{ETCO}_{2} \\
(\mathrm{mmHg})\end{array}$ & Sensitivity & Specificity & PPV & NPV & Accuracy \\
\hline 15 & $100 \%$ & $23 \%$ & $35 \%$ & $100 \%$ & $46 \%$ \\
22 & $100 \%$ & $48 \%$ & $45 \%$ & $100 \%$ & $64 \%$ \\
29 & $100 \%$ & $77 \%$ & $65 \%$ & $100 \%$ & $84 \%$ \\
32 & $100 \%$ & $97 \%$ & $93 \%$ & $100 \%$ & $98 \%$ \\
42 & $92 \%$ & $97 \%$ & $93 \%$ & $100 \%$ & $96 \%$ \\
\hline
\end{tabular}

$\mathrm{ETCO}_{2}$, end-tidal carbon dioxide; NPV, negative predictive value; PPV, positive predictive value; ROSC. return of spontaneous circulation.

a

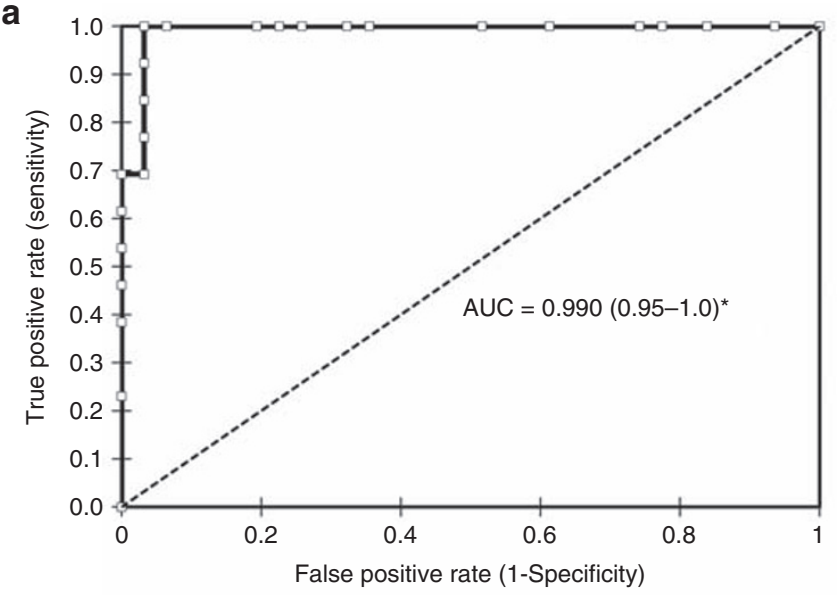

b

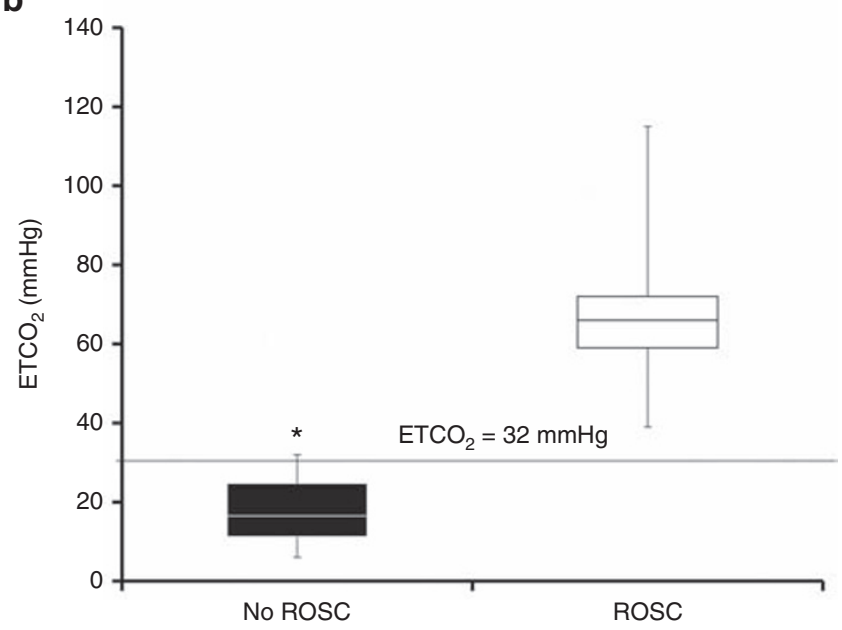

Figure 4. Prediction analysis $\mathrm{ETCO}_{2}$ and ROSC. (a) Receiver operator curve for end-tidal $\mathrm{CO}_{2}\left(\mathrm{ETCO}_{2}\right)$ to predict return of spontaneous circulation (ROSC). (b) Box plots of all ETCO values before and after ROSC. *denotes a statistical significance $P<0.01$.

Prior to achieving ROSC ( $-2 \mathrm{~min}$ and $-1 \mathrm{~min})$, there was no significant difference in $\mathrm{ETCO}_{2}(18.7 \pm 7.5 \mathrm{mmHg}$ and $21.9 \pm 6.9 \mathrm{mmHg}$ respectively, Figure 5a). At ROSC the $\mathrm{ETCO}_{2}$ rapidly increased to $56.8 \pm 19.7 \mathrm{mmHg}$ and was significantly higher than $\mathrm{ETCO}_{2}$ at -1 min prior to $\operatorname{ROSC}(P<0.001)$.

\section{$\mathrm{ETCO}_{2}$ and $\mathrm{PaCO}_{2}$ Correlation Post ROSC}

Analysis of data from 5-10 min after ROSC demonstrated that there was a positive correlation between $\mathrm{ETCO}_{2}$ and 

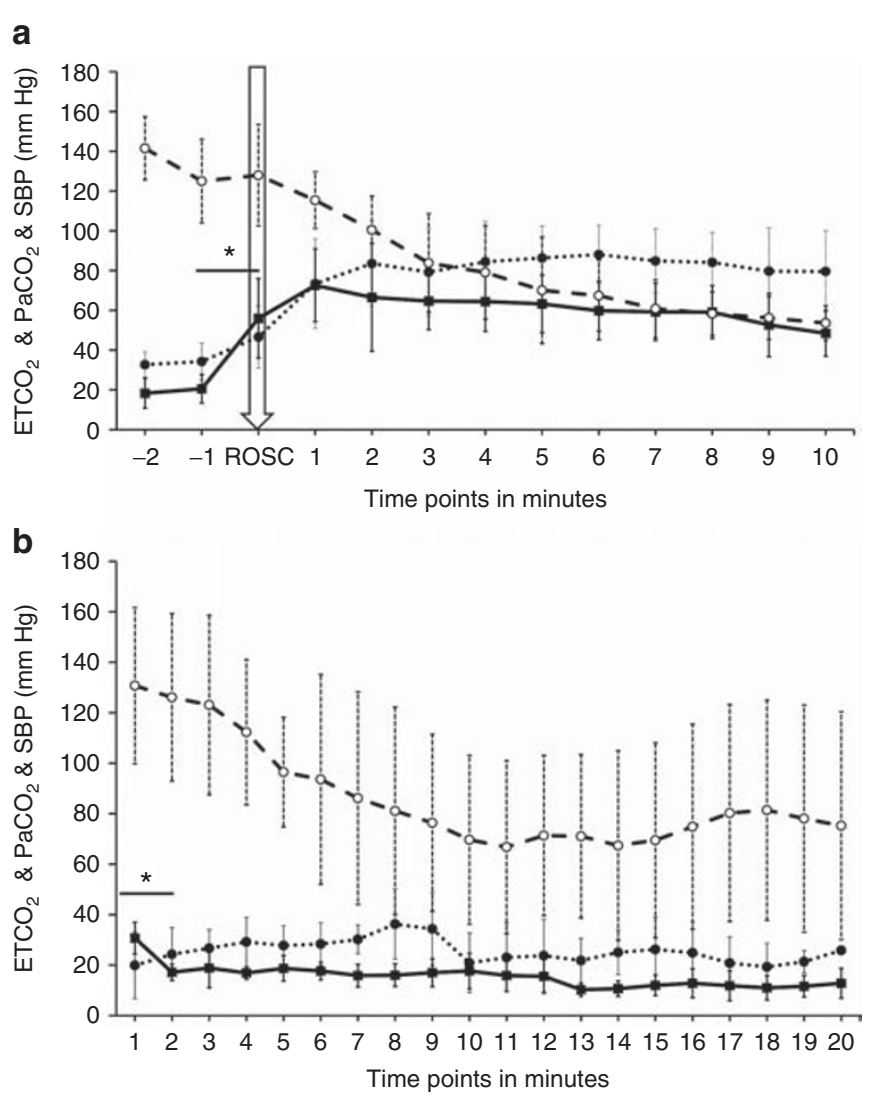

Figure 5. Changes in $\mathrm{ETCO}_{2}, \mathrm{PaCO}_{2^{\prime}}$ and $\mathrm{SBP}$. (a) Changes in $\mathrm{ETCO}_{2}$ (black squares), $\mathrm{PaCO}_{2}$ (white circles) and systolic blood pressure (SBP) (black circles) are shown in lambs that achieved return of spontaneous circulation (ROSC) and (b) in those that did not achieve ROSC. ${ }^{*}$ denotes a statistical significance $P<0.01$.

$\mathrm{PaCO}_{2}$ (157 pairs, $R^{2}=0.64, P<0.001$ ) (Figure 6a). Agreement between $\mathrm{PaCO}_{2}$ and $\mathrm{ETCO}_{2}$ are shown as Bland-Altman plot (Figure 6b). $\mathrm{ETCO}_{2}$ was lower than $\mathrm{PaCO}_{2}$ with a mean and $\mathrm{SD}$ of $-6 \pm 13 \mathrm{mmHg}$.

\section{$\mathrm{ETCO}_{2}$ and $\mathrm{PaCO}_{2}$ in no ROSC}

The $\mathrm{ETCO}_{2}$ and $\mathrm{PaCO}_{2}$ values during the period of resuscitation in lambs that did not achieve ROSC are shown in Figure 5b. The $\mathrm{ETCO}_{2}$ during the first minute of resuscitation was $30.7 \pm 6.3 \mathrm{mmHg}$ and dropped to $17.1 \pm 3.2 \mathrm{mmHg}(P<$ 0.01 ) by second minute and remained below $20 \mathrm{mmHg}$ for the remainder of the resuscitation.

Figure 2a is a graphic depiction of events during resuscitation of a lamb with perinatal cardiac arrest. With initiation of ventilation, there is expulsion of residual $\mathrm{CO}_{2}$ which is seen as a brisk increase in $\mathrm{ETCO}_{2}$ (14). Subsequently, during chest compressions, $\mathrm{ETCO}_{2}$, pulmonary blood flow, and SBP are low. Once ROSC is achieved, there is an abrupt increase in $\mathrm{ETCO}_{2}$ (Figure 2a,b), pulmonary blood flow, and SBP. Figure $\mathbf{2 b}$ shows the events around ROSC. Detectable EKG and rise in $\mathrm{ETCO}_{2}$ at ROSC are shown.

\section{DISCUSSION}

Providing effective chest compressions and PPV without interruption and early detection of ROSC are essential components of neonatal resuscitation. To our knowledge, this is the first study in a lamb model of perinatal asphxial arrest to explore the utility of capnography to assess adequacy of resuscitation and detect ROSC.

Once effective ventilation is established, if the heart rate remains $<60 / \mathrm{min}$, NRP recommends chest compressions to establish circulation, increase intravascular pressures and establish blood flow (5). In the absence of an intra-arterial line, it is difficult to assess the adequacy of chest compressions in the delivery room. In our study, in a fully instrumented lamb, the initiation of chest compressions caused changes in SBP, pulmonary blood flow and $\mathrm{ETCO}_{2}$ which was reflective of effective resuscitation (Figure 2a,b). An increase in SBP appears to be a good marker for effective chest compressions (10). In a previous prospective resuscitation study by Sutton $e t$ al. in children, effective chest compressions resulted in SBP of $\geq 80 \mathrm{mmHg}$ (15). In the same study, for every $10 \mathrm{~mm}$ increase in depth of CC, the average SBP increased by $15 \mathrm{mmHg}$ (15). They also found SBP had the most clinically relevant association with improved rate, depth, and force of chest compressions while the association with diastolic pressures was less clinically robust. In term lambs with baseline fetal systolic pressures of $\sim 60 \mathrm{mmHg}$, and in the presence of diastolic runoff through a patent ductus, we arbitrarily assigned a SBP of $\geq 30 \mathrm{mmHg}$ to define effective chest compressions. This value was approximately half of the fetal baseline SBP. In our study, $\mathrm{ETCO}_{2}$ was able to predict effective SBP ( $\geq 30 \mathrm{mmHg}$ ) with AUC - 0.735 (Figure 3a) with sensitivities ranging from 96 to $69 \%$ for a range of $10-15 \mathrm{mmHg}$ during resuscitation. We understand that diastolic pressure, a more important measure of coronary perfusion, should have been targeted instead of SBP. However, in the presence of a patent ductus arteriosus, diastolic pressures were not an accurate measure as it was associated with reversal of flow.

Many studies involving cardiac arrest models after completion of perinatal transition have shown that $\mathrm{ETCO}_{2}$ is useful in predicting ROSC with high sensitivity and specificity (1618). Chalak et al. have reported an $\mathrm{ETCO}_{2}$ value of $14 \mathrm{mmHg}$ with high sensitivity and specificity to predict ROSC. In this model by Chalak et al., resuscitation was initiated after $1 \mathrm{~min}$ of asystole, while in our study resuscitation was initiated after 5 min of asystole. In our study, in the presence of fetal lung liquid, $\mathrm{ETCO}_{2}$ of $32 \mathrm{mmHg}$ had $100 \%$ sensitivity and near $100 \%$ specificity. Our results were similar to previous animal models $(16,19)$ where resuscitation was initiated 5-10 min after asphyxia. When ROSC is achieved there is a dramatic rise in $\mathrm{ETCO}_{2}$ (Figure 6) and this finding may serve as a useful tool to assess ROSC without interrupting resuscitation in delivery room. In the clinical setting, the importance of $\mathrm{ETCO}_{2}$ during resuscitation has been validated in adults by Falk et al. (20). A retrospective study involving neonates using Pedi-cap a colorimetric $\mathrm{CO}_{2}$ detector has reported color change just prior to ROSC neonatal resuscitation (21) which points to the fact that a rise in $\mathrm{ETCO}_{2}$ regardless of the value can aid in confirming ROSC along with clinical cues without interrupting resuscitation. 


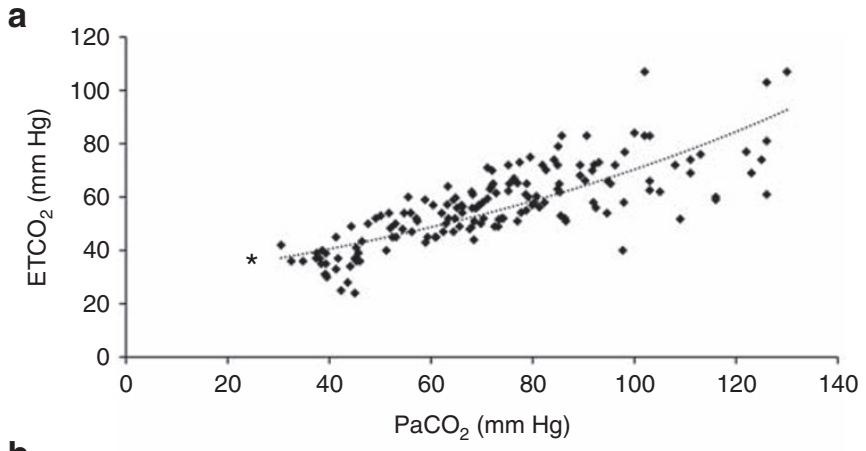

b

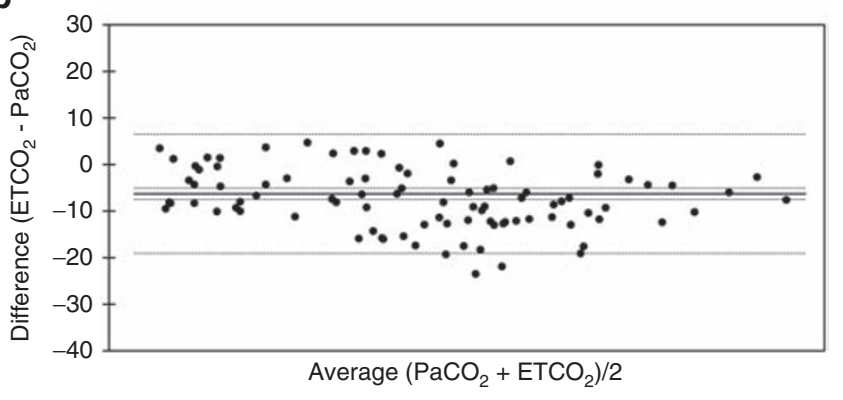

Figure 6. Post resuscitation $\mathrm{ETCO}_{2} \& \mathrm{PaCO}_{2}$. (a) Correlation analysis for $\mathrm{PaCO}_{2}$ and end-tidal $\mathrm{CO}_{2}\left(\mathrm{ETCO}_{2}\right)$ after $5 \mathrm{~min}$ of return of spontaneous circulation (ROSC). 157 pairs (black diamond), ${ }^{*} R^{2}=0.64, P<0.001$. (b) Bland and Altman plot showing the agreement between $\mathrm{PaCO}_{2}$ and $\mathrm{ETCO}_{2}$ (black circles). $\mathrm{ETCO}_{2}$ was lower than $\mathrm{PaCO}_{2}$ with a mean (center dark line) and SD (hyphenated lines at top and bottom) of $-6 \pm 13 \mathrm{mmHg}$.

We have previously shown that $\mathrm{ETCO}_{2}$ correlated well with $\mathrm{PaCO}_{2}$ in an ovine meconium aspiration model poststabilization (11). In this study using an asphyxia model, $\mathrm{ETCO}_{2}$ correlated well with $\mathrm{PaCO}_{2}$ after $5 \mathrm{~min}$ of established ROSC. Infants with asphyxia are at high risk of suffering from associated neurologic injury. Optimal management of these infants after resuscitation includes maintenance of appropriate perfusion, oxygenation and maintaining carbon dioxide levels (13). In these critically ill patients, having a noninvasive feedback device may be helpful to monitor efficacy of resuscitation. While blood gas parameters remain the standard of evaluating gas exchange, $\mathrm{ETCO}_{2}$ monitoring may also serve as a valuable tool in reducing fluctuations in cerebral and pulmonary blood flow, when trended along with intermittent blood gas $\left(\mathrm{PaCO}_{2}\right)$ monitoring.

NRP recently recommended using EKG monitoring during resuscitation to accurately assess heart rate (1). EKG assesses heart rate with higher sensitivity compared with pulse oximetry (22). EKG is likely to be more sensitive in detecting ROSC. However, there are three potential limitations with exclusive use of EKG for detecting ROSC. (i) A case series by Koizumi et al. (23) reported a neonate with pulseless electrical activity with no palpable pulse or heart rate on auscultation. EKG only measures cardiac electrical activity and not perfusion of essential organs. (ii) EKG pattern is difficult to detect during active chest compressions; a brief interruption during chest compressions may be necessary to detect heart rate on EKG (probably during the ventilation period during 3:1 chest compressions to ventilation as recommended by NRP) (Figure 2b). (iii)
Finally, EKG does not assess the adequacy and hemodynamic effectiveness of chest compressions. Hence, we recommend a combination of EKG and $\mathrm{ETCO}_{2}$ monitoring during neonatal resuscitation. The use of multiple devices to detect ROSC is more effective compared with a single feedback device.

A recent study by Li et al. (24) using capnography in a transitioned porcine model has showed significantly better respiratory and hemodynamic parameters during resuscitation in the surviving as compared to nonsurviving piglets. Monitoring $\mathrm{ETCO}_{2}$ and respiratory parameters along with optimal oxygen saturations (25) may be useful during and postresuscitation stabilization.

In interpreting the findings of our study we need to acknowledge several limitations. While we have identified $\mathrm{ETCO}_{2}$ values for predicting SBP of $30 \mathrm{mmHg}$ (roughly half of fetal baseline value) during resuscitation, these are likely to vary by models and may not be true in neonates. Although use of a transitional model is the strength of this study, the presence of a patent ductus may have resulted in diastolic run off during chest compressions. While these findings simulate delivery room resuscitation, it may not reflect resuscitation of an older neonate in the intensive care unit.

\section{Conclusion}

In a large mammalian transitional model of asphyxial cardiac arrest, our study suggests a role for capnography in resuscitation as $\mathrm{ETCO}_{2}$ predicted adequacy of chest compressions as measured by generation of SBP. In addition, a rise in $\mathrm{ETCO}_{2}$ identified ROSC with 100\% sensitivity and 97\% specificity. Post ROSC, $\mathrm{ETCO}_{2}$ correlates well with $\mathrm{PaCO}_{2}$ and may assist in the ongoing management of those who are resuscitated. Future clinical studies monitoring $\mathrm{ETCO}_{2}$ along with EKG may assist in providing uninterrupted resuscitation and efficient detection of ROSC.

\section{STATEMENT OF FINANCIAL SUPPORT}

This work was supported by the Canadian Pediatric Society-Neonatal Resuscitation Program (SL) and 1R01HD072929 (SL), American Academy of Pediatrics-Neonatal Resuscitation Program (MR), and Henry C. and Bertha H. Buswell Fellowship_University at Buffalo-Salary Support (PC).

Disclosure: Authors have no other financial disclosure.

\section{REFERENCES}

1. Perlman JM, Wyllie J, Kattwinkel J, et al.; Neonatal Resuscitation Chapter Collaborators. Part 7: Neonatal Resuscitation: 2015 International Consensus on Cardiopulmonary Resuscitation and Emergency Cardiovascular Care Science With Treatment Recommendations. Circulation 2015;132(16 Suppl 1):S204-41.

2. Perlman JM, Wyllie J, Kattwinkel J, et al.; Neonatal Resuscitation Chapter Collaborators. Part 7: Neonatal Resuscitation: 2015 International Consensus on Cardiopulmonary Resuscitation and Emergency Cardiovascular Care Science With Treatment Recommendations (Reprint). Pediatrics 2015;136 Suppl 2:S120-66.

3. Wyckoff MH, Aziz K, Escobedo MB, et al. Part 13: Neonatal Resuscitation: 2015 American Heart Association Guidelines Update for Cardiopulmonary Resuscitation and Emergency Cardiovascular Care. Circulation 2015;132(18 Suppl 2):S543-60.

4. Wyllie J, Perlman JM, Kattwinkel J, et al.; Neonatal Resuscitation Chapter Collaborators. Part 7: Neonatal Resuscitation: 2015 International Consensus on Cardiopulmonary Resuscitation and Emergency Cardiovascular 


\section{Articles | Chandrasekharan et al.}

Care Science with Treatment Recommendations. Resuscitation 2015;95:e169-201.

5. Wyckoff MH. Neonatal cardiopulmonary resuscitation: critical hemodynamics. NeoReviews 2010;11:123-129.

6. Berg RA, Hemphill R, Abella BS, et al. Part 5: adult basic life support: 2010 American Heart Association Guidelines for cardiopulmonary resuscitation and emergency cardiovascular care. Circulation 2010;122(18 Suppl 3):S685-705.

7. Garza AG, Gratton MC, Salomone JA, Lindholm D, McElroy J, Archer R. Improved patient survival using a modified resuscitation protocol for outof-hospital cardiac arrest. Circulation 2009;119:2597-605.

8. Abella BS, Sandbo N, Vassilatos P, et al. Chest compression rates during cardiopulmonary resuscitation are suboptimal: a prospective study during in-hospital cardiac arrest. Circulation 2005;111:428-34.

9. Cheng A, Hunt EA, Grant D, et al.; International Network for Simulationbased Pediatric Innovation, Research, and Education CPR Investigators. Variability in quality of chest compressions provided during simulated cardiac arrest across nine pediatric institutions. Resuscitation 2015;97: 13-9.

10. Maher KO, Berg RA, Lindsey CW, Simsic J, Mahle WT. Depth of sternal compression and intra-arterial blood pressure during CPR in infants following cardiac surgery. Resuscitation 2009;80:662-4.

11. Chandrasekharan PK, Rawat M, Nair J, et al. Continuous end-tidal carbon dioxide monitoring during resuscitation of asphyxiated term lambs. Neonatology 2016;109:265-73.

12. Rudolph AM, Yuan S. Response of the pulmonary vasculature to hypoxia and $\mathrm{H}+$ ion concentration changes. J Clin Invest 1966;45:399-411.

13. Pappas A, Shankaran S, Laptook AR, et al.; Eunice Kennedy Shriver National Institute of Child Health and Human Development Neonatal Research Network. Hypocarbia and adverse outcome in neonatal hypoxicischemic encephalopathy. J Pediatr 2011;158:752-758.e1.

14. Berg RA, Henry C, Otto CW, et al. Initial end-tidal CO2 is markedly elevated during cardiopulmonary resuscitation after asphyxial cardiac arrest. Pediatr Emerg Care 1996;12:245-8.
15. Sutton RM, French B, Nishisaki A, et al. American Heart Association cardiopulmonary resuscitation quality targets are associated with improved arterial blood pressure during pediatric cardiac arrest. Resuscitation 2013;84:168-72.

16. Bhende MS, Karasic DG, Menegazzi JJ. Evaluation of an end-tidal CO2 detector during cardiopulmonary resuscitation in a canine model for pediatric cardiac arrest. Pediatr Emerg Care 1995;11:365-8.

17. Chalak LF, Barber CA, Hynan L, Garcia D, Christie L, Wyckoff MH. End-tidal $\mathrm{CO}_{2}$ detection of an audible heart rate during neonatal cardiopulmonary resuscitation after asystole in asphyxiated piglets. Pediatr Res 2011;69(5 Pt 1):401-5.

18. Hamrick JL, Hamrick JT, Lee JK, Lee BH, Koehler RC, Shaffner DH. Efficacy of chest compressions directed by end-tidal $\mathrm{CO}_{2}$ feedback in a pediatric resuscitation model of basic life support. J Am Heart Assoc 2014;3:e000450.

19. Bhende MS, Karasic DG, Karasic RB. End-tidal carbon dioxide changes during cardiopulmonary resuscitation after experimental asphyxial cardiac arrest. Am J Emerg Med 1996;14:349-50.

20. Falk JL, Rackow EC, Weil MH. End-tidal carbon dioxide concentration during cardiopulmonary resuscitation. N Engl J Med 1988;318:607-11.

21. Blank D, Rich W, Leone T, Garey D, Finer N. Pedi-cap color change precedes a significant increase in heart rate during neonatal resuscitation. Resuscitation 2014;85:1568-72.

22. Katheria A, Rich W, Finer N. Electrocardiogram provides a continuous heart rate faster than oximetry during neonatal resuscitation. Pediatrics 2012;130:e1177-81.

23. Koizumi M, Mizumoto H, Araki R, Kan H, Akashi R, Hata D. The utility of electrocardiogram for evaluation of clinical cardiac arrest in neonatal resuscitation. Resuscitation 2016;104:e3-4.

24. Li ES, Cheung PY, O'Reilly M, et al. Exhaled $\mathrm{CO}_{2}$ parameters as a tool to assess ventilation-perfusion mismatching during neonatal resuscitation in a Swine model of neonatal asphyxia. PLoS One 2016;11:e0146524.

25. Lakshminrusimha S, Swartz DD, Gugino SF, et al. Oxygen concentration and pulmonary hemodynamics in newborn lambs with pulmonary hypertension. Pediatr Res 2009;66:539-44. 